\title{
Plasma Expansion into a Vacuum with an Arbitrarily Oriented External Magnetic Field.
}

36th International Workshop on High Density Energy Physics with Intense Ion and Laser Beams.

Hirschegg, Austria.

F. García Rubio $^{1, *}$, A. Ruocco ${ }^{2} \&$ J. Sanz ${ }^{1}$

${ }^{1}$ E.T.S.I.A.E. Universidad Politéctina de Madrid 28040, Madrid.

${ }^{2}$ Universitá degli Studi di Napoli Federico II 80138 Napoli, Italy.

* fernando.garcia.rubio@upm.es

3rd February, 2016 
1. Introduction

2 Problem Statement

- Self-Similarity Hypothesis

3 Perpendicular Magnetic Field Case

- Effect of the Magnetic Field Intensity

4 Oblique Magnetic Field Case

- Recovery of Perpendicular Case

5 Conclusions 


\section{Index}

11 Introduction

22. Problem Statement

-

31 Perpendicular Magnetic Field Case

n- Effect of the Magnetic Field Intensity

14 Oblique Magnetic Field Case

- Recovery of Perpendicular Case

5. Conclusions 


\section{Motivation}

- Inertial Confinement Fusion (ICF)

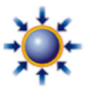

1) Atmosphere formation

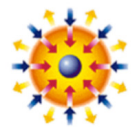

2) Compression

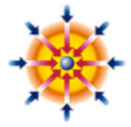

3) Ignition

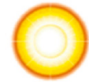

4) Burn
Blowoff $\rightarrow$ Inward transported thermal energy
- Magneto Inertial Fusion (MIF)

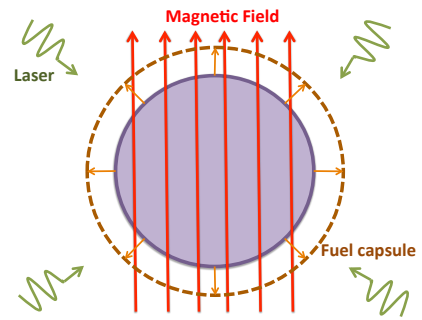

- Astrophysics ${ }^{1}$

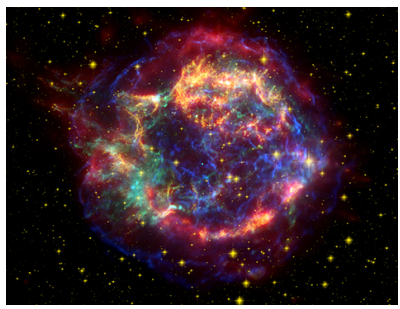

${ }^{1}$ Picture source:

http://www.space.com/11425-photos-supernovas-starexplosions.html 


\section{Previous Work}

\section{Magnetised plasma expansion}

- Ideal magnetohydrodynamics framework.

- Perpendicular magnetic field.

- Inconsistencies when obtaining a complete self-similar solution.

$\checkmark$ D. Anderson, M. Bonnnedal and M. Lisak, "Effect of magnetic field on self-similar plasma expansion into vacuum", Phys. Scr. 22, 507-509, (1980).

$\checkmark$ D. Bennaceur-Doumaz and M. Djebli, "Effect of transverse magnetic field on laser produced plasma expansion into vacuum", Phys. Plasmas 18, 084507 (2011). 


\section{Novelties}

$\checkmark$ Complete self-similar magnetised plasma expansion against a magnetised vacuum.

$\checkmark$ Arbitrarily oriented external magnetic field. 


\section{Index}

\section{Introduction}

2 Problem Statement

- Self-Similarity Hypothesis

[3] Perpendicular Magnetic Field Case

- Effect of the Magnetic Field Intensity

(4) Oblique Magnetic Field Case

- Recovery of Perpendicular Case

5. Conclusions 


\section{Governing Equations}

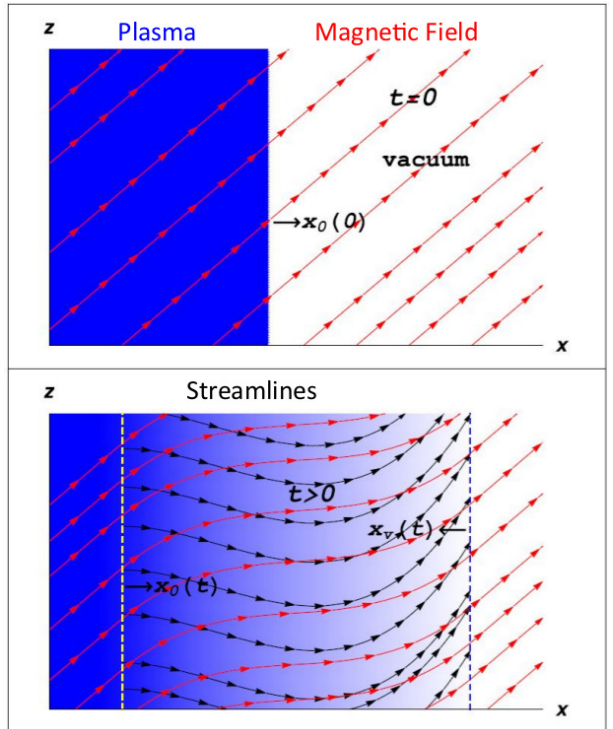




\section{Governing Equations}

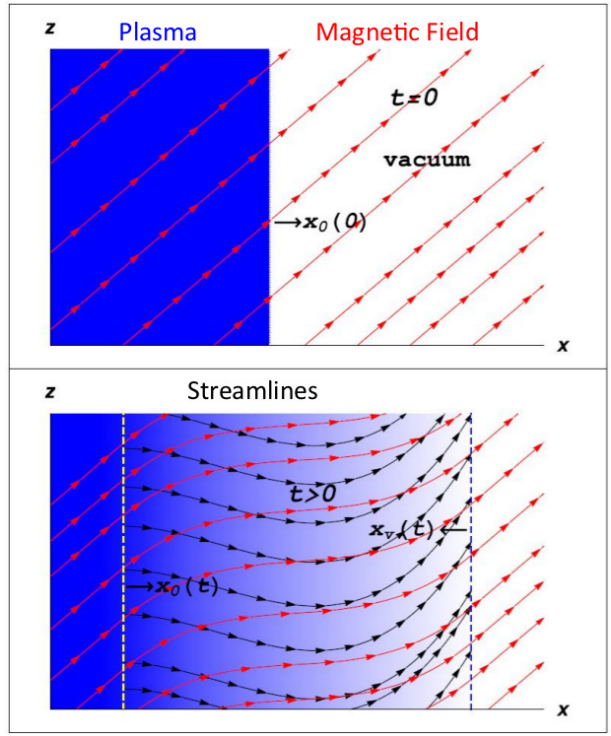

\section{Fluid equations}

$$
\frac{\partial \rho}{\partial t}+\nabla \cdot(\rho \vec{V})=0,
$$

$$
\begin{gathered}
\rho \frac{\partial \vec{V}}{\partial t}+\rho \vec{V} \cdot \nabla \vec{V}+\frac{1}{\mu_{0}} \vec{B} \times(\nabla \times \vec{B})+\nabla p=0 \\
p=p_{0}\left(\frac{\rho}{\rho_{0}}\right)^{\gamma} .
\end{gathered}
$$

\section{Maxwell's equations}

$$
\begin{gathered}
\frac{\partial \vec{B}}{\partial t}-\nabla \times(\vec{V} \times \vec{B})=0, \\
\nabla \cdot \vec{B}=0 .
\end{gathered}
$$




\section{Boundary Conditions (I)}

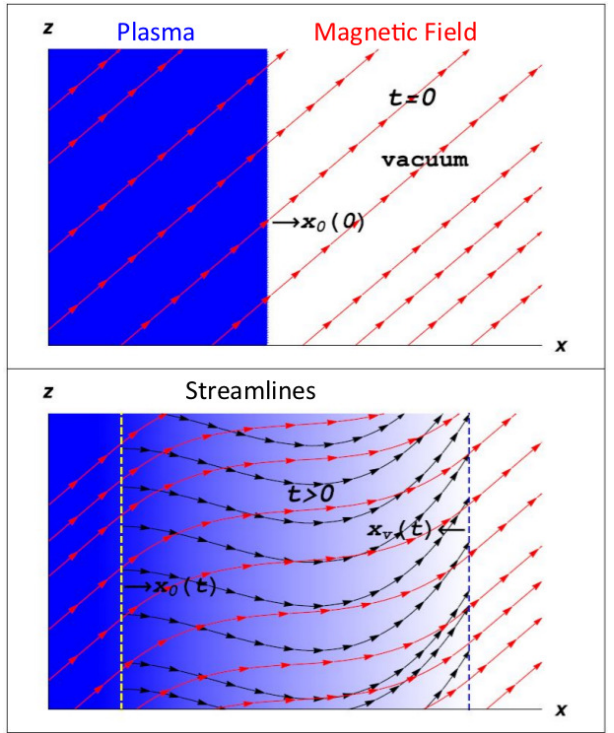

Boundary Condition at $x \rightarrow-\infty$

$\checkmark$ Recovery of the unperturbed plasma.

$\checkmark$ Frontier $x=x_{0}(t)$ travelling at constant velocity towards the unperturbed plasma.

$\checkmark$ None perturbation for $x<x_{0}(t)$. 


\section{Boundary Conditions (II)}

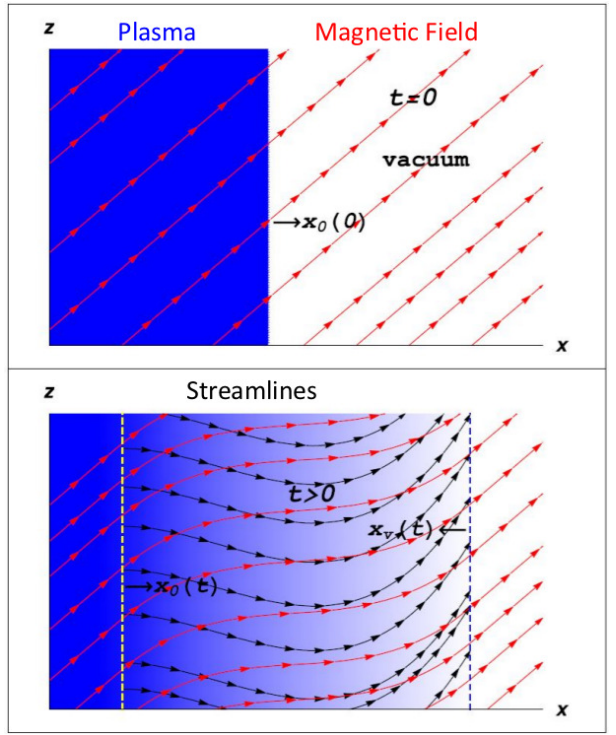

\section{Border with the Vacuum}

$\checkmark$ Frontier $x=x_{v}(t)$ travelling at constant velocity towards $x>0$.

$\checkmark$ It is a fluid surface:

$$
V_{x}\left(t, x=x_{v}\right)=\mathrm{d} x_{v}(t) / \mathrm{d} t .
$$

$\checkmark$ Momentum conservation yields jump conditions. 


\section{Self-Similarity and Dimensionless Equations}

$\checkmark$ No characteristic length $\rightarrow$ Dependence on $\xi=x /\left(c_{0} t\right)$.

$\checkmark$ Dimensionless variables:

$$
n(\xi)=\rho / \rho_{0}, \quad b_{z}(\xi)=B_{z} / B_{0}, \quad u(\xi)=V_{x} / c_{0}, \quad w(\xi)=V_{z} / c_{0} .
$$




\section{Self-Similarity and Dimensionless Equations}

$\checkmark$ No characteristic length $\rightarrow$ Dependence on $\xi=x /\left(c_{0} t\right)$.

$\checkmark$ Dimensionless variables:

$$
n(\xi)=\rho / \rho_{0}, \quad b_{z}(\xi)=B_{z} / B_{0}, \quad u(\xi)=V_{x} / c_{0}, \quad w(\xi)=V_{z} / c_{0} .
$$

Dimensionless governing equations

$$
\begin{gathered}
(u-\xi) n^{\prime}+n u^{\prime}=0, \\
n(u-\xi) u^{\prime}+n^{\gamma-1} n^{\prime}+\frac{\left(b_{z}^{2}\right)^{\prime}}{\beta}=0, \\
n(u-\xi) w^{\prime}-\frac{2 \cos \alpha}{\beta} b_{z}^{\prime}=0, \\
(u-\xi) b_{z}^{\prime}+b_{z} u^{\prime}-w^{\prime} \cos \alpha=0 .
\end{gathered}
$$




\section{Self-Similarity and Dimensionless Equations}

$\checkmark$ No characteristic length $\rightarrow$ Dependence on $\xi=x /\left(c_{0} t\right)$.

$\checkmark$ Dimensionless variables:

$$
n(\xi)=\rho / \rho_{0}, \quad b_{z}(\xi)=B_{z} / B_{0}, \quad u(\xi)=V_{x} / c_{0}, \quad w(\xi)=V_{z} / c_{0} .
$$

Dimensionless governing equations

$$
\begin{gathered}
(u-\xi) n^{\prime}+n u^{\prime}=0, \\
n(u-\xi) u^{\prime}+n^{\gamma-1} n^{\prime}+\frac{\left(b_{z}^{2}\right)^{\prime}}{\beta}=0, \\
n(u-\xi) w^{\prime}-\frac{2 \cos \alpha}{\beta} b_{z}^{\prime}=0, \\
(u-\xi) b_{z}^{\prime}+b_{z} u^{\prime}-w^{\prime} \cos \alpha=0 .
\end{gathered}
$$

\section{Type of solutions}

$\checkmark$ Trivial solution: plateau.

$$
n, u, w, b_{z}=\text { const. }
$$

$\checkmark$ Non-linear waves: Dispersion relation.

$$
f\left(n, u, w, b_{z} ; \xi\right)=0 .
$$




\section{Self-Similarity and Dimensionless Equations}

$\checkmark$ No characteristic length $\rightarrow$ Dependence on $\xi=x /\left(c_{0} t\right)$.

$\checkmark$ Dimensionless variables:

$$
n(\xi)=\rho / \rho_{0}, \quad b_{z}(\xi)=B_{z} / B_{0}, \quad u(\xi)=V_{x} / c_{0}, \quad w(\xi)=V_{z} / c_{0} .
$$

Dimensionless governing equations

$$
\begin{gathered}
(u-\xi) n^{\prime}+n u^{\prime}=0, \\
n(u-\xi) u^{\prime}+n^{\gamma-1} n^{\prime}+\frac{\left(b_{z}^{2}\right)^{\prime}}{\beta}=0, \\
n(u-\xi) w^{\prime}-\frac{2 \cos \alpha}{\beta} b_{z}^{\prime}=0, \\
(u-\xi) b_{z}^{\prime}+b_{z} u^{\prime}-w^{\prime} \cos \alpha=0 .
\end{gathered}
$$

\section{Type of solutions}

$\checkmark$ Trivial solution: plateau.

$$
n, u, w, b_{z}=\text { const. }
$$

$\checkmark$ Non-linear waves: Dispersion relation.

$$
f\left(n, u, w, b_{z} ; \xi\right)=0 .
$$

Governing parameters

$\alpha=$ Initial magnetic field inclination

$$
\beta=\frac{2 \gamma \mu_{0} p_{0}}{B_{0}^{2}}=2\left(\frac{c_{0}}{v_{A 0}}\right)^{2}
$$




\section{Index}

3 Perpendicular Magnetic Field Case

- Effect of the Magnetic Field Intensity

4 Oblique Magnetic Field Case

- Recovery of Perpendicular Case

5. Conclusions 


\section{Dispersion Relation}

$$
\alpha=\frac{\pi}{2}, B_{x}=0, w=0
$$

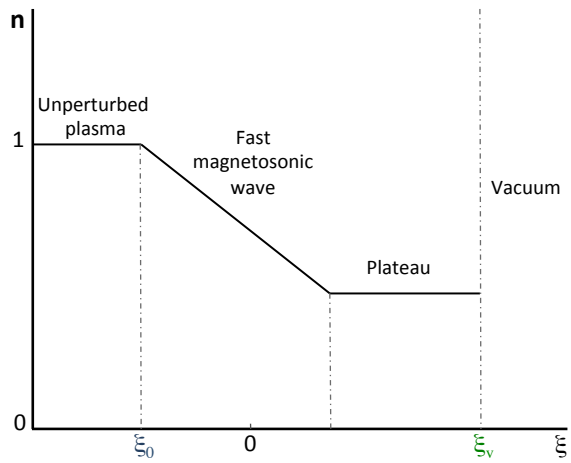

$\checkmark$ Dispersion relation: Magnetosonic wave.

$$
(u-\xi)^{2}=n^{\gamma-1}+2 \frac{b_{z}^{2}}{n \beta},
$$




\section{Dispersion Relation}

$$
\alpha=\frac{\pi}{2}, B_{x}=0, w=0
$$

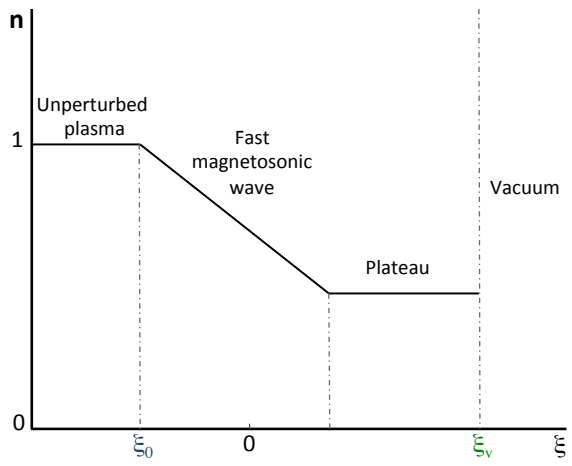

$\checkmark$ Dispersion relation: Magnetosonic wave.

$$
\begin{gathered}
(u-\xi)^{2}=n^{\gamma-1}+2 \frac{b_{z}^{2}}{n \beta}, \\
(u-\xi)^{2}=\left(\frac{c}{c_{0}}\right)^{2}+\left(\frac{v_{A}}{c_{0}}\right)^{2} .
\end{gathered}
$$




\section{Dispersion Relation}

$$
\alpha=\frac{\pi}{2}, B_{x}=0, w=0
$$

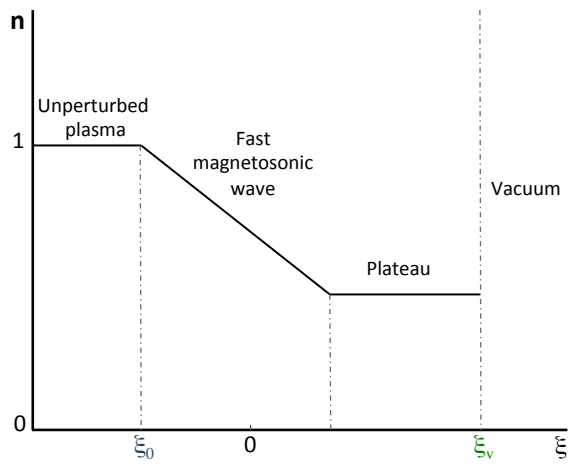

$\checkmark$ Dispersion relation: Magnetosonic wave.

$$
\begin{gathered}
(u-\xi)^{2}=n^{\gamma-1}+2 \frac{b_{z}^{2}}{n \beta}, \\
(u-\xi)^{2}=\left(\frac{c}{c_{0}}\right)^{2}+\left(\frac{v_{A}}{c_{0}}\right)^{2} .
\end{gathered}
$$

$\checkmark$ The unperturbed plasma can be retrieved by the magnetosonic wave.

$$
\xi_{0}=-\sqrt{1+\frac{2}{\beta}} .
$$




\section{Dispersion Relation}

$$
\alpha=\frac{\pi}{2}, B_{x}=0, w=0
$$

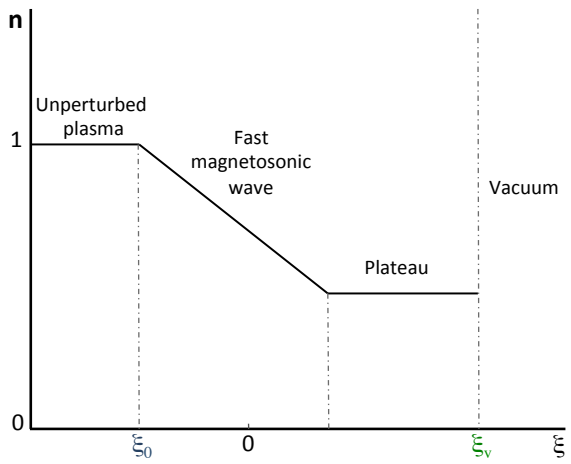

$\checkmark$ Dispersion relation: Magnetosonic wave.

$$
\begin{gathered}
(u-\xi)^{2}=n^{\gamma-1}+2 \frac{b_{z}^{2}}{n \beta}, \\
(u-\xi)^{2}=\left(\frac{c}{c_{0}}\right)^{2}+\left(\frac{v_{A}}{c_{0}}\right)^{2} .
\end{gathered}
$$

$\checkmark$ The unperturbed plasma can be retrieved by the magnetosonic wave.

$$
\xi_{0}=-\sqrt{1+\frac{2}{\beta}} .
$$

$\checkmark$ The frontier with the vacuum cannot be retrieved by the magnetosonic wave. 


\section{Solution for $\gamma=5 / 3$}

Plasma Expansion with Perpendicular Magnetic Field. $\beta=10, \gamma=5 / 3$.

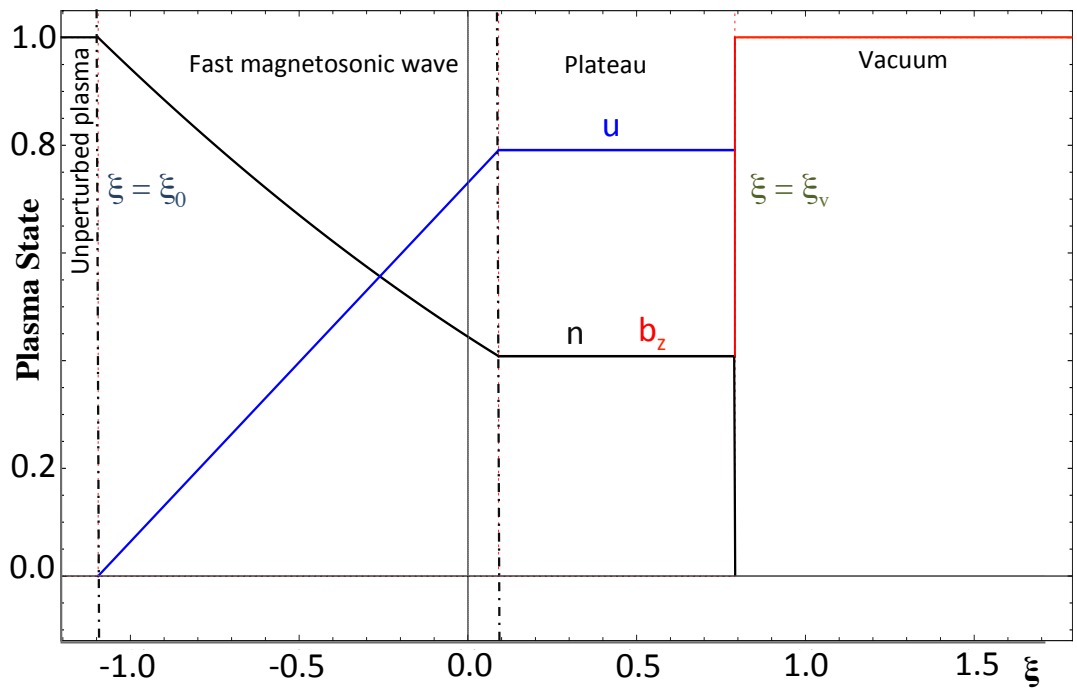


Features of the expansion depending on $\beta$

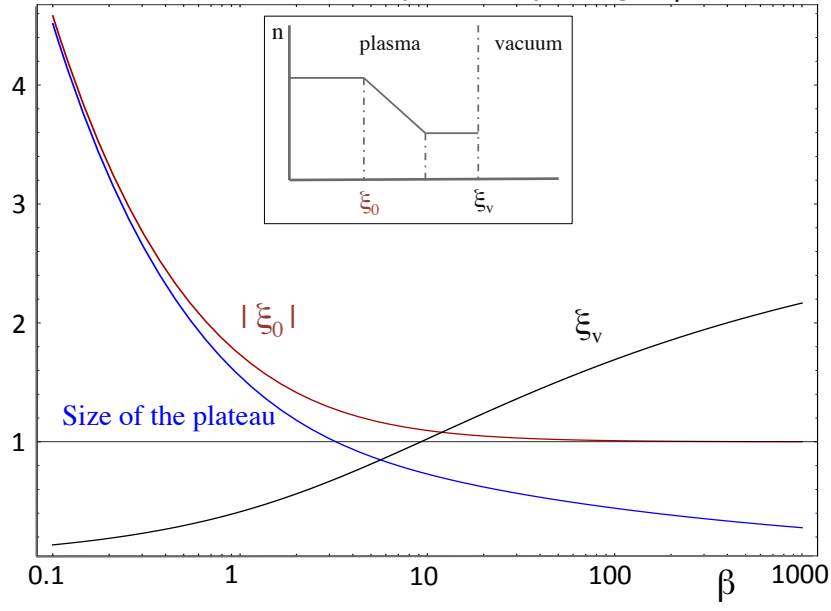

$\beta \ll 1$, Strong magnetic field $\mid \beta \gg 1$, Weak magnetic field High confinement

Standard fluid 


\section{Index}

4 Oblique Magnetic Field Case

- Recovery of Perpendicular Case 


\section{Dispersion Relation}

$$
\alpha<\frac{\pi}{2}, B_{x}=\text { const } \neq 0, w \neq 0
$$

$$
(u-\xi)^{2}=\frac{1}{2}\left(\frac{c}{c_{0}}\right)^{2}+\frac{1}{2}\left(\frac{v_{A}}{c_{0}}\right)^{2} \pm \frac{1}{2} \sqrt{\left[\left(\frac{c}{c_{0}}\right)^{2}+\left(\frac{v_{A}}{c_{0}}\right)^{2}\right]^{2}-4\left(\frac{c}{c_{0}}\right)^{2}\left(\frac{v_{A}}{c_{0}}\right)^{2} \cos ^{2} \theta}
$$

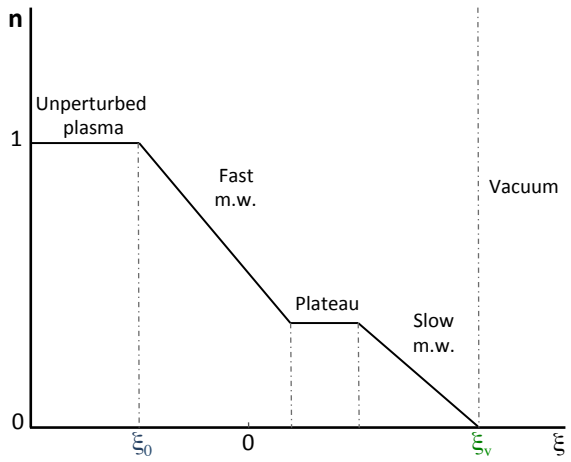




\section{Dispersion Relation}

$$
\alpha<\frac{\pi}{2}, B_{x}=\text { const } \neq 0, w \neq 0
$$

$$
(u-\xi)^{2}=\frac{1}{2}\left(\frac{c}{c_{0}}\right)^{2}+\frac{1}{2}\left(\frac{v_{A}}{c_{0}}\right)^{2} \pm \frac{1}{2} \sqrt{\left[\left(\frac{c}{c_{0}}\right)^{2}+\left(\frac{v_{A}}{c_{0}}\right)^{2}\right]^{2}-4\left(\frac{c}{c_{0}}\right)^{2}\left(\frac{v_{A}}{c_{0}}\right)^{2} \cos ^{2} \theta}
$$

Fast Magnetosonic Wave.

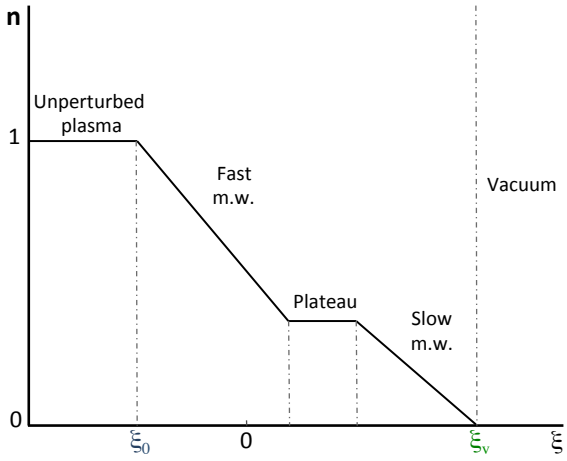

$\checkmark$ Sign + ,

$\checkmark$ Connects with $\xi=\xi_{0}$. 


\section{Dispersion Relation}

$$
\alpha<\frac{\pi}{2}, B_{x}=\text { const } \neq 0, w \neq 0
$$

$$
(u-\xi)^{2}=\frac{1}{2}\left(\frac{c}{c_{0}}\right)^{2}+\frac{1}{2}\left(\frac{v_{A}}{c_{0}}\right)^{2} \pm \frac{1}{2} \sqrt{\left[\left(\frac{c}{c_{0}}\right)^{2}+\left(\frac{v_{A}}{c_{0}}\right)^{2}\right]^{2}-4\left(\frac{c}{c_{0}}\right)^{2}\left(\frac{v_{A}}{c_{0}}\right)^{2} \cos ^{2} \theta}
$$

Fast Magnetosonic Wave.

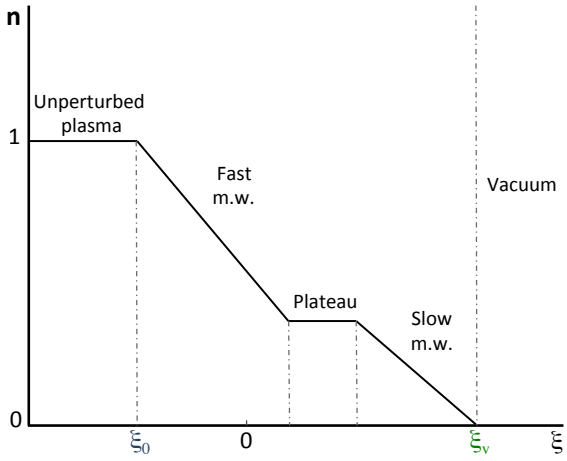

$\checkmark$ Sign + ,

$\checkmark$ Connects with $\xi=\xi_{0}$.

Slow Magnetosonic Wave.

$\checkmark$ Sign -,

$\checkmark$ Connects with $\xi=\xi_{v}$,

$\checkmark$ Weak discontinuity. 


\section{Solution for $\gamma=5 / 3$}

Plasma Expansion with Oblique Magnetic Field. $\alpha=\pi / 4, \beta=10, \gamma=5 / 3$

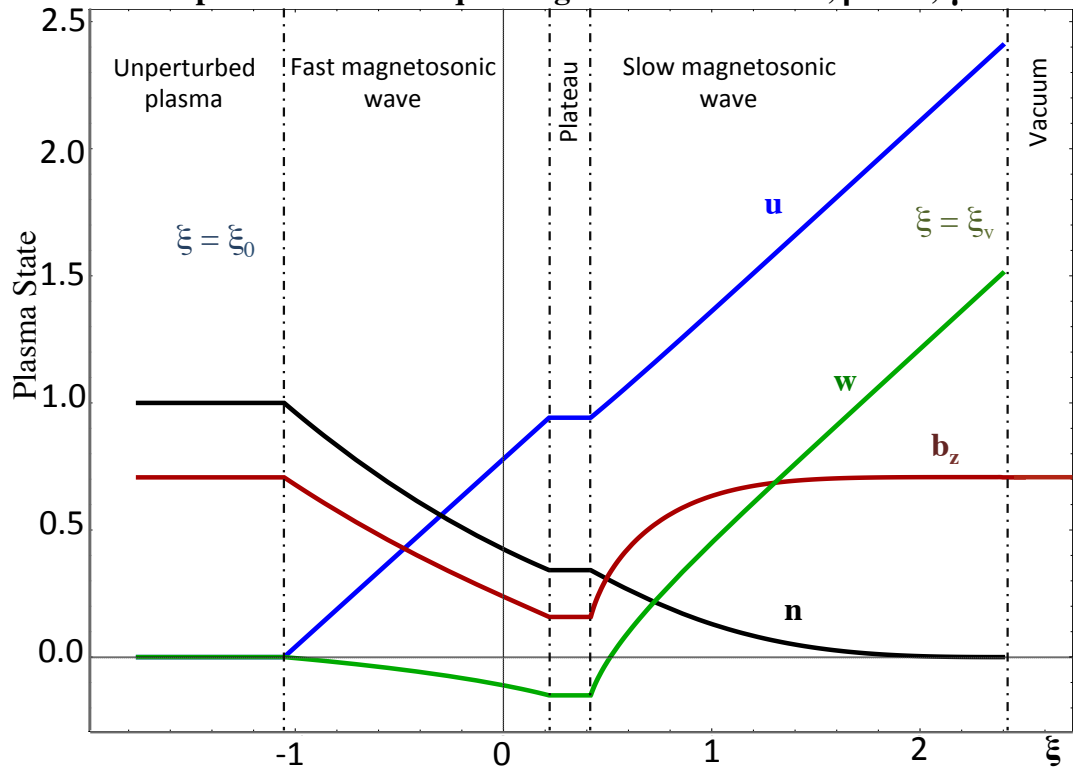




\section{Effect of the Inclination of the Magnetic Field Lines}

Velocity of the wave entering the unperturbed plasma. $\gamma=5 / 3$

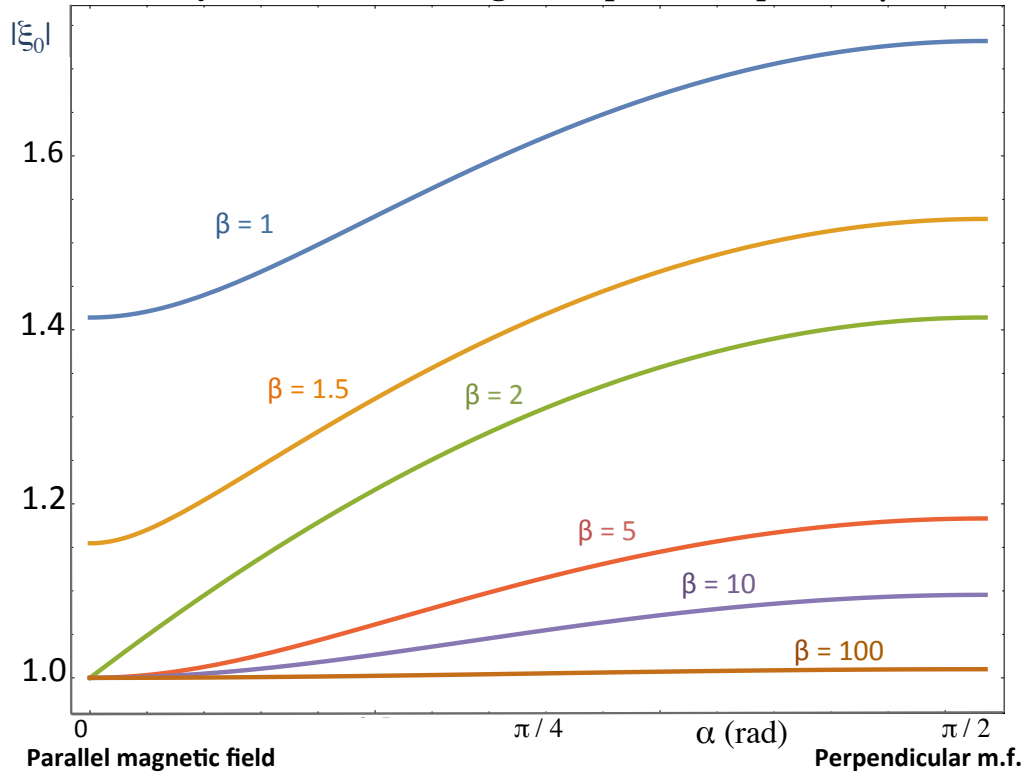




\section{Recovery of the Standard Fluid Expansion, $\beta>2$}

$$
\beta=2\left(\frac{c_{0}}{v_{A 0}}\right)^{2}
$$
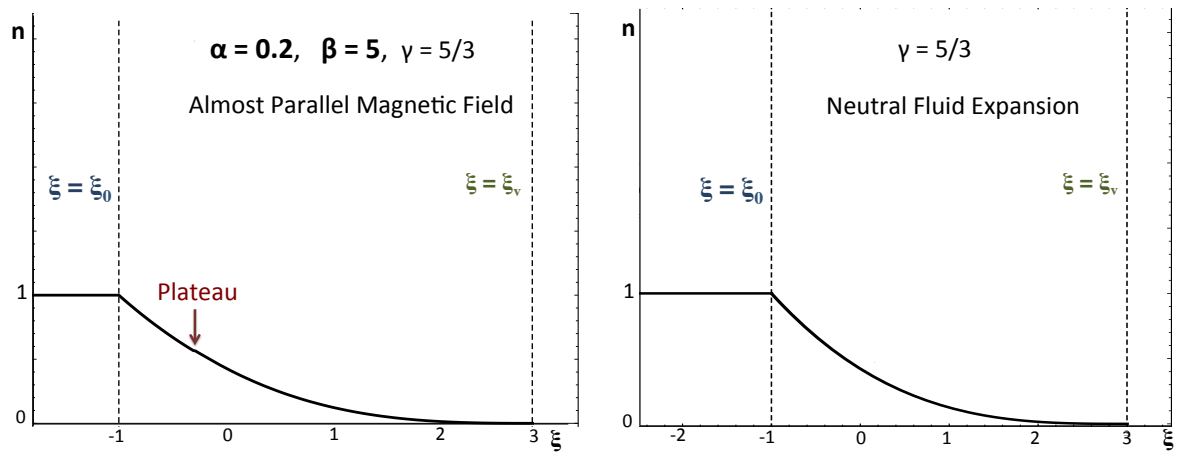


\section{Recovery of the Standard Fluid Expansion, $\beta<2$}

$$
\beta=2\left(\frac{c_{0}}{v_{A 0}}\right)^{2}
$$
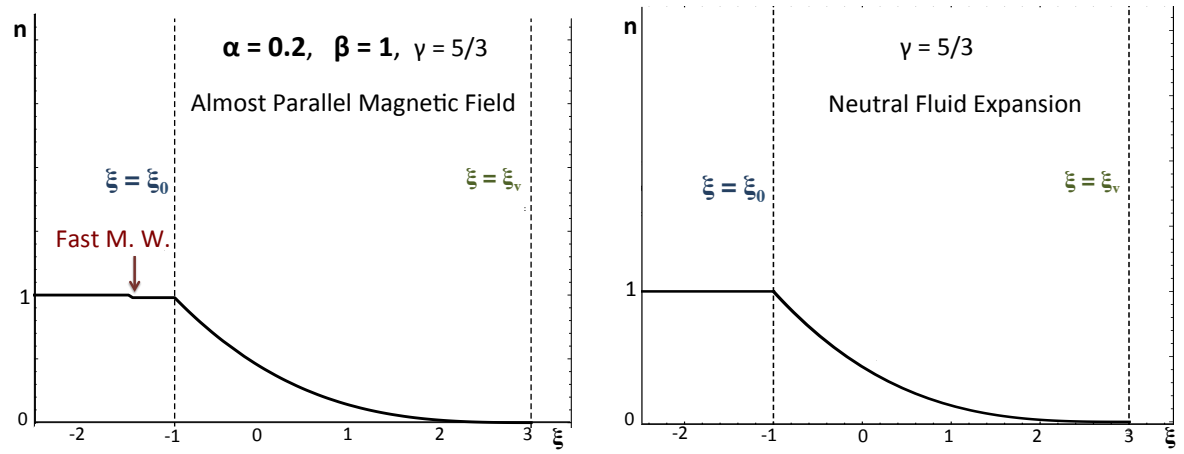


\section{Recovery of the Perpendicular Magnetic Field Expansion}

$$
|\alpha-\pi / 2|=\eta \ll 1 \quad \xi_{v}-\xi_{2}=\mathcal{O}\left(\eta^{2}\right)
$$

Plasma Expansion with Oblique Magnetic Field. $\alpha=\pi / 2-0.15, \beta=10, \gamma=2$

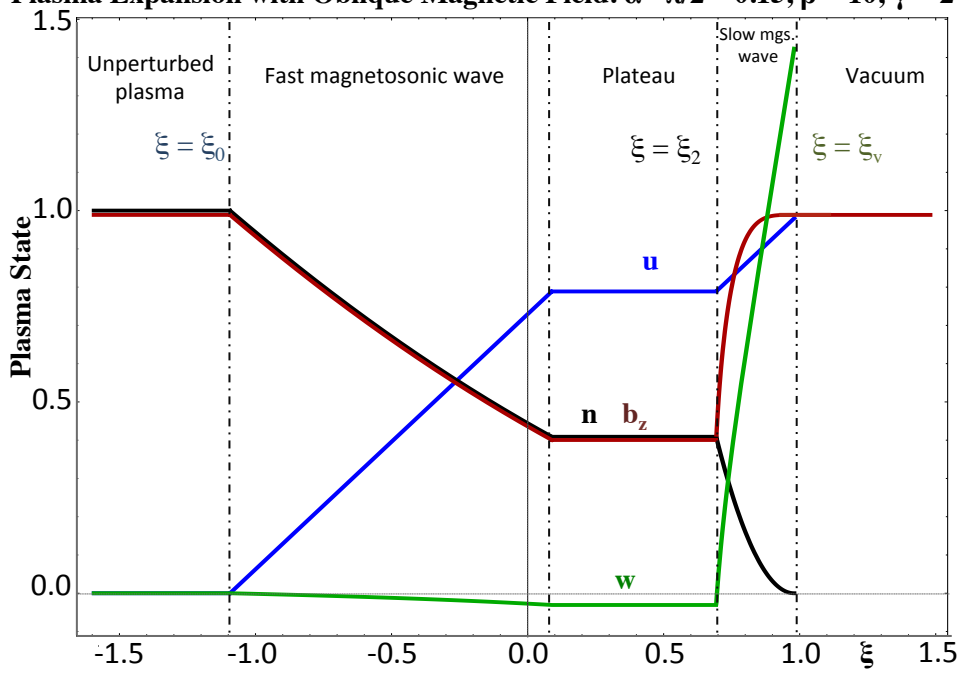




\section{Index}

II Introduction

2. Problem Statement

- Self-Similarity Hypothesis

13. Perpendicular Magnetic Field Case

- Effect of the Magnetic Field Intensity

4 Oblique Magnetic Field Case

- Recovery of Perpendicular Case

5. Conclusions 


\section{Conclusions}

$\checkmark$ Plasma expansion into vacuum with an external magnetic field is a self-similar process.

$\checkmark$ Solution: piecewise matching non-linear waves and plateaus. 


\section{Conclusions}

$\checkmark$ Plasma expansion into vacuum with an external magnetic field is a self-similar process.

$\checkmark$ Solution: piecewise matching non-linear waves and plateaus.

$\checkmark$ Perpendicular magnetic field $\rightarrow$ Density discontinuity at the edge of the expansion. 
$\checkmark$ Plasma expansion into vacuum with an external magnetic field is a self-similar process.

$\checkmark$ Solution: piecewise matching non-linear waves and plateaus.

$\checkmark$ Perpendicular magnetic field $\rightarrow$ Density discontinuity at the edge of the expansion.

$\checkmark$ Oblique magnetic field $\rightarrow$ Weak discontinuity at the edge of the expansion. 
$\checkmark$ Plasma expansion into vacuum with an external magnetic field is a self-similar process.

$\checkmark$ Solution: piecewise matching non-linear waves and plateaus.

$\checkmark$ Perpendicular magnetic field $\rightarrow$ Density discontinuity at the edge of the expansion.

$\checkmark$ Oblique magnetic field $\rightarrow$ Weak discontinuity at the edge of the expansion.

$\checkmark$ The net effect of the magnetic field is to confine the expansion. 
$\checkmark$ Plasma expansion into vacuum with an external magnetic field is a self-similar process.

$\checkmark$ Solution: piecewise matching non-linear waves and plateaus.

$\checkmark$ Perpendicular magnetic field $\rightarrow$ Density discontinuity at the edge of the expansion.

$\checkmark$ Oblique magnetic field $\rightarrow$ Weak discontinuity at the edge of the expansion.

$\checkmark$ The net effect of the magnetic field is to confine the expansion.

$\checkmark$ Serve as test case for M.H.D. code validation.

F. García Rubio, A. Ruocco, J. Sanz, "Plasma expansion into a vacuum with an arbitrarily oriented external magnetic field". Phys, Plasmas 23, 012103 (2016). 


\section{THANK YOU FOR YOUR ATTENTION}

Any questions? 


\section{Boundary Conditions}

Recovery of the unperturbed plasma at $x \rightarrow-\infty$ :

$\checkmark$ Frontier $x=x_{0}(t)$ travelling at constant velocity towards the unperturbed plasma.

$\checkmark$ None perturbation for $x<x_{0}(t)$.

\section{Border with the vacuum}

$\checkmark$ Frontier $x=x_{v}(t)$ travelling at constant velocity towards $x>0$.

$\checkmark$ It is a fluid surface:

$$
V_{x}\left(t, x=x_{v}\right)=\mathrm{d} x_{v}(t) / \mathrm{d} t .
$$

$\checkmark$ Momentum conservation impose:

$$
\begin{gathered}
p_{0}\left(\frac{\rho\left(t, x_{v}\right)}{\rho_{0}}\right)^{\gamma}+\frac{B_{z}^{2}\left(t, x_{v}\right)}{2 \mu_{0}}=\frac{B_{0}^{2} \sin ^{2} \alpha}{2 \mu_{0}}, \\
\cos \alpha\left(B_{z}\left(t, x_{v}\right)-B_{0} \sin \alpha\right)=0 .
\end{gathered}
$$

$\checkmark$ Perpendicular magnetic field $(\alpha=\pi / 2) \rightarrow$ Density jump.

$\checkmark$ Oblique magnetic field $(\alpha<\pi / 2) \rightarrow$ Weak discontinuity, $\rho\left(t, \xi_{v}\right)=0$. 


\section{Asymptotic Expressions}

Strong magnetic field $\beta \ll 1$

$$
\begin{gathered}
n_{p}=1-\frac{\beta}{\gamma}+\mathcal{O}\left(\beta^{2}\right), \\
\xi_{0}=-\sqrt{\frac{2}{\beta}}+\mathcal{O}\left(\beta^{1 / 2}\right), \\
\xi_{v}=\frac{1}{\gamma} \sqrt{\frac{\beta}{2}}+\mathcal{O}\left(\beta^{3 / 2}\right) .
\end{gathered}
$$

Weak magnetic field $\beta \gg 1$

$$
\begin{gathered}
n_{p}=\left(\frac{\gamma}{\beta}\right)^{1 / \gamma}+\mathcal{O}\left(\beta^{-3 / \gamma}\right), \\
\xi_{0}=-1-\frac{1}{\beta}+\mathcal{O}\left(\beta^{-2}\right), \\
\xi_{v}=\frac{2}{\gamma-1}\left[1-\left(\frac{\gamma}{\beta}\right)^{(\gamma-1) /(2 \gamma)}\right]+\mathcal{O}\left(\beta^{-1}\right) .
\end{gathered}
$$




\section{Analytical Solution for $\gamma=2$}

$\checkmark$ When $\gamma=2$, the system of governing equations is analytically integrable.

$\checkmark$ Magnetosonic wave structure:

$$
\begin{gathered}
u=2 \sqrt{1+\frac{2}{\beta}}(1-\sqrt{n}) . \\
\left.b_{z}=n \text { (also true for a general } \gamma\right),
\end{gathered}
$$

$\checkmark$ Plasma state at the plateau, its foot and end:

$$
\begin{gathered}
n_{p}=b_{z p}=\sqrt{\frac{2 / \beta}{1+2 / \beta}}, \\
u_{p}=\xi_{v}=2 \sqrt{1+2 / \beta}\left(1-\sqrt[4]{\frac{2 / \beta}{1+2 / \beta}}\right), \\
\xi_{1}=2 \sqrt{1+2 / \beta}\left(1-\frac{3}{2} \sqrt[4]{\frac{2 / \beta}{1+2 / \beta}}\right) .
\end{gathered}
$$




\section{Recovery of the Standard Fluid Expansion}

$$
\beta>2
$$
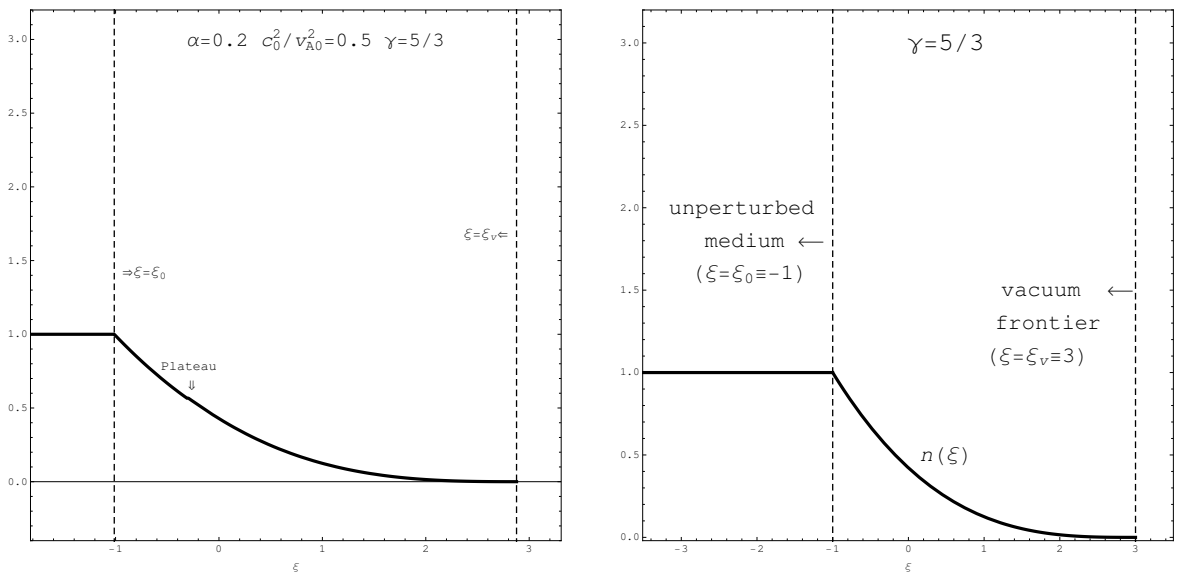


\section{Recovery of the Standard Fluid Expansion}
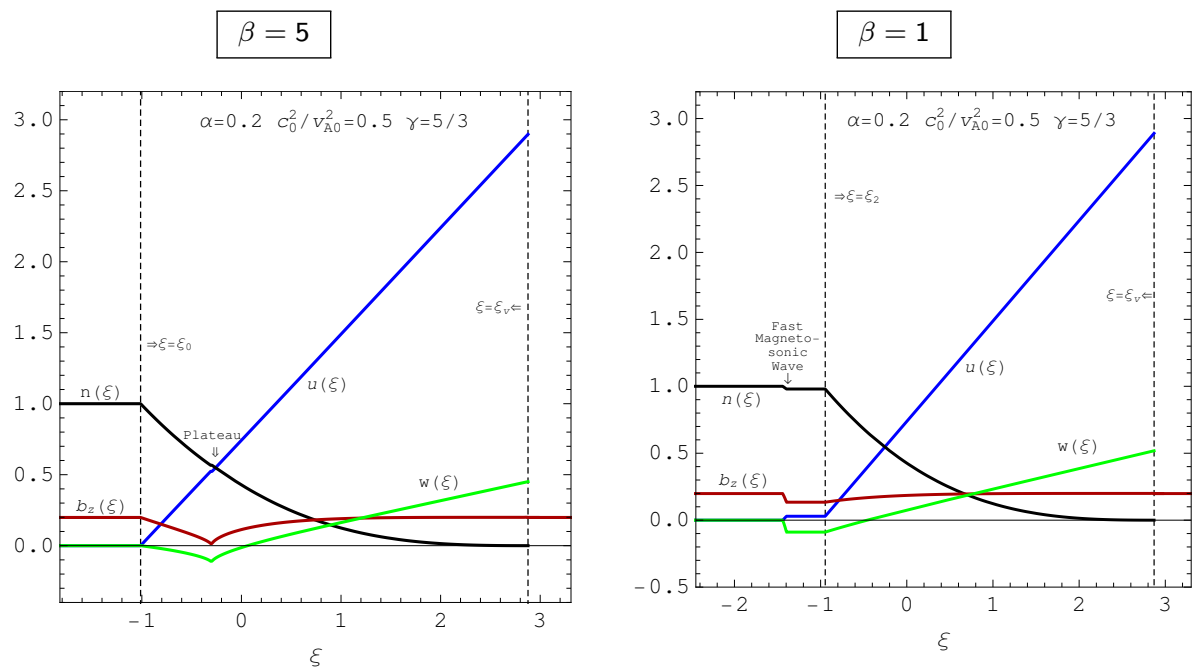